\title{
IL-37 expression is decreased in patients with hyperhomocysteinemia and protects cells from inflammatory injury by homocysteine
}

\author{
SEN WANG ${ }^{1 *}$, ZHEN HUANG ${ }^{2 *}$, WENRUI LI ${ }^{3 *}$, SUHUI HE $^{1}$, HONGMEI WU ${ }^{2}$, \\ JIANDONG ZHU $^{1}$, RUMENG LI ${ }^{1}$, ZHUANGYAN LIANG ${ }^{1}$ and ZHANGQUAN CHEN ${ }^{1}$ \\ ${ }^{1}$ Key Laboratory for Medical Molecular Diagnostics of Guangdong Province, Guangdong Medical University, \\ Dongguan, Guangdong 523808; ${ }^{2}$ Longgang Central Hospital, Shenzhen, Guangdong 518116; \\ ${ }^{3}$ Eighth People's Hospital of Dongguan City, Dongguan, Guangdong 523321, P.R. China
}

Received March 31, 2019; Accepted September 4, 2019

DOI: $10.3892 / \mathrm{mmr} .2019 .10804$

\begin{abstract}
As a novel anti-inflammatory cytokine of the interleukin (IL)-1 family, IL-37 protects the human body from diseases characterized by excessive inflammation. The pathologic process of hyperhomocysteinemia (hHcy) is accompanied by persistent inflammation. However, little is known regarding the role of IL-37 in hHcy. In the present study, the levels of cytokines including IL-37, IL-1 $\beta$, IL-6 and tumor necrosis factor- $\alpha$ in the supernatant were detected by ELISA. mRNA and protein expression were detected by Reverse transcription-quantitative PCR and western blotting, respectively. $\mathrm{LDH}$ level was determined by ELISA and the cell viability was detected through $\mathrm{CCK}-8 \mathrm{kit}$. In the present study, mean serum IL-37 levels of patients with hHcy were $32.3 \%$ lower than those of controls $(\mathrm{P}<0.01)$. In peripheral blood mononuclear cells (PBMCs) from patients with hHcy, mean IL-37 mRNA expression was $73.5 \%$ lower $(\mathrm{P}<0.01)$ and IL-37 protein expression was $77.7 \%$ lower compared with that of healthy controls $(\mathrm{P}<0.01)$. Furthermore, the results demonstrated that exogenous homocysteine (Hcy) stimulation markedly downregulated the mRNA and protein expression levels of IL-37 in PBMCs in vitro. In 293T cells,
\end{abstract}

Correspondence to: Dr Zhuangyan Liang or Dr Zhangquan Chen, Key Laboratory for Medical Molecular Diagnostics of Guangdong Province, Guangdong Medical University, 1 Xincheng Road, Dongguan, Guangdong 523808, P.R. China

E-mail: czqhk@126.com

E-mail: zqchen@gdmu.edu.cn

${ }^{*}$ Contributed equally

Abbreviations: CCK-8, Cell Counting Kit-8; eGFP, enhanced green fluorescent protein; Hcy, homocysteine; hHcy, hyperhomocysteinemia; IL-37, interleukin 37; LDH, lactate dehydrogenase; PBMCs, peripheral blood mononuclear cells

Key words: IL-37, expression, hHcy, Hcy, inflammation overexpression of IL-37 restored the cell viability impaired by Hcy, and reduced the release of lactate dehydrogenase and the proinflammatory cytokines IL-1 $\beta$, IL-6 and tumor necrosis factor- $\alpha$. In conclusion, IL-37 was downregulated by Hcy in vivo and in vitro, and IL-37 exhibited a protective role against cell injury induced by Hcy.

\section{Introduction}

Homocysteine (Hcy), a sulfur-containing amino acid, is a key intermediate product in the metabolism of methionine $(1,2)$. Hyperhomocysteinemia ( $\mathrm{hHcy}$ ) is an independent risk factor for vascular diseases, and results in cumulative lesions in cardiovascular (1-4), renal $(5,6)$ and brain tissues (7-10). Clinically, a Hcy level exceeding $15 \mu \mathrm{mol} / \mathrm{l}$ is regarded as hHcy (11-14). Elevated Hcy acts as an independent risk factor for vascular diseases (1-4). Previous studies have revealed that hHcy is involved in a number of clinical diseases, including cardiovascular disease (1-4), stroke (7,8), Alzheimer's disease (10) and kidney disease $(5,6)$. For example, Rong et al (3) reported that the risk of peripheral arterial disease is associated with serum Hcy levels independent of classical vascular risk factors. Elevated Hcy causes blood vessel damage, which results in inflammation and plaque formation $(3,4)$. High blood pressure with high Hcy levels has become a serious public health problem (1-4).

Interleukin (IL)-37 is one of the newest members of the interleukin family, and may be a novel medicine for the interventional treatment of vascular diseases according to previous studies (15-25). IL-37 is a natural innate immune inhibitor that inhibits the expression of a variety of inflammatory factors (15-17). IL-37 is highly expressed in the blood of patients with autoimmune diseases, including inflammatory bowel disease (18), rheumatoid arthritis $(19,20)$ and systemic lupus erythematosus (21). Additionally, elevated levels of IL-37 have been detected in the blood of patients with atherosclerosis (22), acute coronary syndromes (23), myocardial infarction and cerebral ischemia (24). Furthermore, animal experiments have demonstrated that IL-37 improves myocardial function after myocardial death, and reduces inflammatory responses after 
cerebral ischemia and reperfusion (24). In another study, IL-37 could be utilized in the treatment of atherosclerosis (22). In addition, increased IL-37 expression has been demonstrated in most diseases, whereas decreased IL-37 expression has been found in only a few diseases, including Vogt-Koyanagi-Harada disease (25).

As an important novel molecule involved in inflammatory responses, the expression and biological function of IL-37 in hHcy remain unknown. The present study revealed that the expression levels of IL-37 were decreased in the sera and peripheral blood mononuclear cells (PBMCs) of patients with hHcy, and overexpression of IL-37 could reverse Hcy-induced cell damage and inflammatory response.

\section{Materials and methods}

Materials. Hcy was purchased from Sigma-Aldrich and used at a concentration of $20-80 \mu \mathrm{mol} / 1$ to treat cells for $24 \mathrm{~h}$. Anti-IL-37 monoclonal antibody (ab153889) and anti-human $\beta$-actin monoclonal antibody (ab6276) were obtained from Abcam. Ficoll separation liquid was purchased from Pharmacia Biotech. The eukaryotic expression vector for IL-37 fused with the enhanced green fluorescent protein (eGFP; pcDNA3.1/IL-37-eGFP) was constructed in our laboratory. Hydrophilic PVDF membranes were purchased from EMD Millipore. The Reverse Transcript kit [PrimeScript ${ }^{\mathrm{TM}}$ RT reagent Kit (Perfect Real Time)] and PCR kit [TB Green ${ }^{\circledR}$ Premix Ex Taq ${ }^{\mathrm{TM}}$ II (Tli RNaseH Plus)] were obtained from Takara Biotechnology Co., Ltd.. FBS and DMEM were purchased from Gibco; Thermo Fisher Scientific, Inc. ELISA kits IL-37 (cat. no. 88-52103-22, Thermo Fisher Scientific, Inc.), IL-6 (cat. no. BMS213-2, Thermo Fisher Scientific, Inc.), IL-1 $\beta$ (cat. no. BMS224-2, Thermo Fisher Scientific, Inc.), TNF- $\alpha$ (cat. no. 88-7346-22, Thermo Fisher Scientific, Inc.) for cytokines and Lipofectamine ${ }^{\circledR} 2000$ transfection reagent were obtained from Thermo Fisher Scientific, Inc. Lactate dehydrogenase (LDH) testing kits (cat. no. A020-2-2) were purchased from Nanjing Jiancheng Bioengineering Institute. The Cell Counting Kit-8 (CCK-8) was obtained from Beyotime Institute of Biotechnology.

Specimens. The experiments were approved by the Science and Education Section of Eighth People's Hospital of Dongguan City, which is responsible for ethical approval at the Eighth People's Hospital of Dongguan City. Written informed consent was obtained from all participants for the use of their samples in the present study. A total of 47 patients with hHcy were recruited at the Eighth People's Hospital of Dongguan City between September 2017 and December 2017. Patients with severe hyperhomocysteinemia usually have a variety of serious complications, and these complications are likely to affect the level of IL-37, meaning the effect of pure homocysteine on IL-37 would be difficult to detect. Therefore, the present study mainly chose patients with mild hHcy. Additionally, 35 healthy controls were recruited. Case screening was based on the internationally recognized definitions of hHcy (11-14). The age of patients ranged between 22 and 82 years, and 20 and 55 years for healthy controls. The average age of patients with hHcy and controls was $52.36 \pm 6.658$ and $49.29 \pm 7.434$ years, respectively. There were 30 males and 17 females in the patients and
24 males and 11 females in the healthy controls. In the present study, the inclusion criteria included: i) Subjects with normal clinical biochemical indicators; and ii) the level of Hcy in serum was $>15 \mu \mathrm{mol} / 1$. Subjects with abnormal liver and kidney function, and those with tumors, diabetes and autoimmune diseases were excluded. Patients who were receiving drugs that could influence Hcy metabolism were also excluded. Patients with abnormal lipid and carbohydrate metabolism, or with immune system diseases were also excluded.

Cell culture. PBMCs were isolated from fresh bloods of both the patients and healthy controls using Ficoll separation liquid (Beijing Dingguo Changsheng Biotechnology Co., Ltd.), according to the manufacturer's protocol $(500 \mathrm{x} \mathrm{g}$ at room temperature for $20 \mathrm{~min}$ ). The purity was checked under a light microscope, and was identified as $>95 \%$. The supernatant was also collected. The PBMCs were collected and suspended in $1 \mathrm{ml}$ RPMI-1640 medium (Gibco; Thermo Fisher Scientific, Inc.). The cells $\left(6 \times 10^{5}\right.$ cells $\left./ \mathrm{ml}\right)$ were added to 24 -well plates and were placed in a $\mathrm{CO}_{2}$ incubator at $37^{\circ} \mathrm{C}$ for $2-4 \mathrm{~h}$. When the cells grew well without blistering, shrinkage or abnormal secretory granules, they were collected for subsequent experiments. The 293T cell line was purchased from The Cell Bank of Type Culture Collection of the Chinese Academy of Sciences. Cells were cultured in DMEM containing 10\% FBS, $100 \mathrm{U} / \mathrm{ml}$ penicillin and $100 \mathrm{~g} / \mathrm{ml}$ streptomycin. The culture conditions were $37^{\circ} \mathrm{C}$ and $5 \% \mathrm{CO}_{2}$.

Reverse transcription-quantitative PCR (RT-qPCR). Total RNA from PBMCs $\left(1 \times 10^{6}\right.$ cells $)$ or $293 \mathrm{~T}$ cells $\left(1 \times 10^{6}\right.$ cells/well $)$ was extracted according to the instructions of the RNA Isolation kit (Takara Biotechnology Co., Ltd.). Subsequently, cDNA was synthesized using a cDNA Synthesis kit [PrimeScript ${ }^{\mathrm{TM}}$ RT reagent Kit (Perfect Real Time), Takara Biotechnology Co., Ltd.]. The RNA was reversely transcripted to DNA in a reaction as follows: 5X PrimeScript RT Master Mix, $2 \mu \mathrm{l}$; Total RNA, $500 \mathrm{ng}$, and then RNasefree $\mathrm{ddH}_{2} \mathrm{O}$ added to $10 \mu \mathrm{l}$. The temperature protocol was: $65^{\circ} \mathrm{C} 5 \mathrm{~min}, 42^{\circ} \mathrm{C} 30 \mathrm{~min}, 70^{\circ} \mathrm{C}$ $15 \mathrm{~min}$ and then retained at $4^{\circ} \mathrm{C}$. qPCR was carried out using a SYBR $^{\circledR}$ Premix Ex Taq ${ }^{\mathrm{TM}}$ kit (Takara Biotechnology Co., Ltd.) on an ABI Prism 7700 Sequence Detection system (Applied Biosystems; Thermo Fisher Scientific, Inc.). A total of $25 \mu 1$ reaction buffer was prepared (12.5 $\mu 12$ X SYBR Green Master Mix, $300 \mathrm{nM}$ primer and $1 \mu \mathrm{l} \mathrm{cDNA})$. The conditions for $\mathrm{qPCR}$ were: $30 \mathrm{sec}$ at $95^{\circ} \mathrm{C}$, followed by 40 cycles of $5 \mathrm{sec}$ at $95^{\circ} \mathrm{C}$ and $34 \mathrm{sec}$ at $60^{\circ} \mathrm{C}$ and $30 \mathrm{sec}$ at $72^{\circ} \mathrm{C}$. Melt curve stage: $15 \mathrm{sec}$ at $95^{\circ} \mathrm{C}$ and $60 \mathrm{sec}$ at $60^{\circ} \mathrm{C}$ and $15 \mathrm{sec}$ at $95^{\circ} \mathrm{C}$. Finally, the samples were kept at $4^{\circ} \mathrm{C}$. The primers were synthesized by Sangon Biotech Co., Ltd. Primers for IL-37 were as follows: Forward, 5'-TTAGAAGACCCGGCTGGAAGCC-3' and reverse, 5'-AGATCTCTGGGCGTATGTAGT-3'. Primers for GAPDH were: Forward: 5'-GAAGGTCGGAGTCAACGG ATTT-3', reverse: 5'-CCTGGAAGATGGTGATGGGATT-3'. Each experiment was performed in triplicate. The average value of the replicates was used as the quantitative value for each sample. The relative mRNA levels were calculated and normalized using the $2^{-\Delta \Delta \mathrm{Cq}}$ method relative to GAPDH (26).

Western blotting. Total proteins of PBMCs were extracted using RIPA buffer ( $1 \mathrm{ml} /$ well, Thermo Fisher Scientific, Inc.) 
and the concentration of the proteins was determined by Enhanced BCA protein assay kit (P0010, Beyotime Institute of Biotechnology). Proteins (50 $\mu \mathrm{g} /$ lane) were separated via SDS-PAGE (6\% concentrated gel and $12 \%$ separation gel) and transferred onto a PVDF membrane for antibody incubation. The blocking reagent was $5 \%$ non-fat milk $(1 \mathrm{~h}$ at room temperature). The primary antibody specific for IL-37 was then diluted at 1:500 to incubate the membrane overnight at $4^{\circ} \mathrm{C}$. The following day, after the membrane was washed by TBS with $0.1 \%$ Tween 20 , the membrane was incubated with secondary antibody (D110087, BBI life sciences) conjugated with horseradish peroxidase $(1: 1,000)$ for $1 \mathrm{~h}$ at room temperature. The protein bands on the membrane were detected using Beyo ECL Star (P0018AS, Beyotime Institute of Biotechnology) by the chemiluminescence method. The results were analyzed using Quantity One software (v 462_PC, Bio-Rad Laboratories, Inc.).

Gene transfection. In the present study, IL-37 was overexpressed by transfecting pcDNA3.1/IL-37-eGFP plasmid into $293 \mathrm{~T}$ cells, while cells of the control group were transfected with empty pcDNA3.1 vector. 293T Cells $\left(1 \times 10^{5}\right.$ cells $\left./ \mathrm{ml}\right)$ were cultured in 24-well plates prior to gene transfection. Lipofectamine $^{\circledR} 2000(1.5 \mu \mathrm{l})$ and $400 \mathrm{ng}$ DNA were mixed and added into wells (50 $\mu \mathrm{l} /$ well) for gene transfection. Subsequently, the cells were cultured with $5 \% \mathrm{CO}_{2}$ at $37^{\circ} \mathrm{C}$ prior to RNA and protein extraction. RNA was extracted after $1,3,6$, or $9 \mathrm{~h}$ and protein was extracted after $20 \mathrm{~h}$.

ELISA. This experiment was performed according to the manufacturer's protocol of the ELISA kit. Each specimen was tested in three wells. The samples were treated using tetramethylbenzidine color liquid, and the absorbance was determined at $450 \mathrm{~nm}$ with an enzyme analyzer. The concentration of the sample to be measured was calculated according to the absorbance, and the formula was obtained from the standard curve.

$L D H$ assay. LDH assay was performed according to the kit instructions. Cells in the control group were treated with PBS and cells of the experimental group were treated with Hcy. Briefly, cells were treated with different concentrations of Hcy $(0,30,60$ or $120 \mu \mathrm{mol} / \mathrm{l})$. Then the supernatants were collected for LDH detection. The experimental wells were added with $20 \mu \mathrm{l}$ samples, $25 \mu \mathrm{l}$ basic buffer and $5 \mu \mathrm{l}$ coenzyme 1 , while $5 \mu$ double steamed water was used to replace the coenzyme in the blank wells. The liquids were mixed and incubated at $37^{\circ} \mathrm{C}$ for $15 \mathrm{~min}$. Then, $25 \mu 12$,4-dinitrophenylhydrazine and $0.4 \mathrm{~mol} / \mathrm{l} \mathrm{NaOH}$ were added and allowed to stand still for $5 \mathrm{~min}$. The absorbance of the solution was measured at $450 \mathrm{~nm}$ using the iMark microplate Reader (Bio-Rad Laboratories, Inc.). LDH levels in the media were quantified and compared to control values according to the instructions of the kit.

Cell viability. Cells were cultured in a 6 -well plate at $37^{\circ} \mathrm{C}$ with $5 \% \mathrm{CO}_{2}$ and were digested with trypsin for collection. Subsequently, 5,000 cells/well were seeded in 96-well plates. For each group, five wells were used. The absorbance at $450 \mathrm{~nm}$ was tested at 24, 48 and $72 \mathrm{~h}$ using CCK-8. The CCK-8 assay was performed according to the manufacturer's protocol, 5,000 cells were seeded into a well of the 96-well plate for $24 \mathrm{~h}$ incubation in a humidified incubator $\left(37^{\circ} \mathrm{C}, 5 \%\right.$ $\left.\mathrm{CO}_{2}\right)$. Following treatment with $\mathrm{Hcy}$ at various concentrations $(0-80 \mu \mathrm{mol} / 1$ for PBMCs and 30, 60, $120 \mu \mathrm{mol} / \mathrm{l}$ for 293T cells at $37^{\circ} \mathrm{C}$ ) for another $24 \mathrm{~h}, 10 \mu \mathrm{l} \mathrm{CCK}-8$ reagent were added to each well of the plate for $2 \mathrm{~h}$ at $37^{\circ} \mathrm{C}$ in the incubator. Cells in the control group were treated with PBS. The absorbance was measured at $450 \mathrm{~nm}$ using a microplate reader. All experiments were performed in triplicate and repeated three independent times.

Statistical analysis. All experiments were performed in triplicate and repeated three independent times. Data were analyzed using SPSS v19.0 software (IBM Corp.) and presented as the mean \pm SD. The Bonferroni method was used to compare independent samples between two groups. Multivariate ANOVA (one-way) was used. $\mathrm{P}<0.05$ was considered to indicate a statistically significant difference.

\section{Results}

IL-37 levels are decreased in the sera and PBMCs of patients with $h H c y$. The control group included healthy individuals and the experimental group included patients with high Hcy. The mean concentrations of Hcy for patients and controls were $21.10 \pm 6.57$ and $11.11 \pm 2.74 \mu \mathrm{mol} / 1$, respectively (Fig. 1A). ELISA results demonstrated that the IL-37 levels of patients with hHcy were $32.3 \%$ lower than those of the controls ( $\mathrm{P}=0.0022$; Fig. 1A). Furthermore, in PBMCs, IL-37 mRNA expression was $73.5 \%$ lower compared with the controls $(\mathrm{P}<0.0001$; Fig. 1B). In Fig. 1C, the blood samples $1-7$ were selected mainly according to the mean Hcy levels of patients and controls mentioned above. Individuals whose Hcy concentration was close to the average concentration were selected ( $21.10 \mu \mathrm{mol} / 1$ for patients and $11.11 \mu \mathrm{mol} / 1$ for controls). The IL-37 protein expression in PBMCs of patients with mild hHcy was $77.7 \%$ lower than that of the controls (Fig. 1C).

IL-37 expression is downregulated by Hcy in PBMCs. To further investigate why IL-37 expression was downregulated in patients with hHcy, the effects of Hcy on IL-37 expression were investigated. In this experiment, cells of the control group were treated with PBS. In the experimental group, PBMCs were treated with Hcy (20-80 $\mu \mathrm{mol} / \mathrm{l})$ for $1,3,6$ and $9 \mathrm{~h}$ to extract total RNAs and $24 \mathrm{~h}$ to extract total proteins. qPCR results demonstrated that IL-37 mRNA expression was significantly reduced. The IL-37 mRNA expression was reduced by $77.0 \%$ by $20 \mu \mathrm{mol} / 1 \mathrm{Hcy}$, compared with the control group ( $\mathrm{P}<0.01$; Fig. 2A). IL-37 protein expression was significantly reduced following Hcy treatment. The IL-37 protein expression was reduced by $84.3 \%$ by $20 \mu \mathrm{mol} / 1 \mathrm{Hcy}$ compared with the control group $(\mathrm{P}<0.01$; Fig. $2 \mathrm{~B})$.

Exogenous Hcy inhibits cell viability, and induces LDH and proinflammatory cytokines in 2937 cells. The biological functions of IL-37 were further determined. First, the effects of Hcy on cells were investigated. After 293T cells were stimulated by Hcy $(30,60$ and $120 \mu \mathrm{mol} / \mathrm{l})$ for $24 \mathrm{~h}$, a CCK- 8 assay was performed to measure cell viability. Cells in the control group were treated with PBS. Cell viability was inhibited by higher concentrations of Hcy. There was no significant inhibitory 

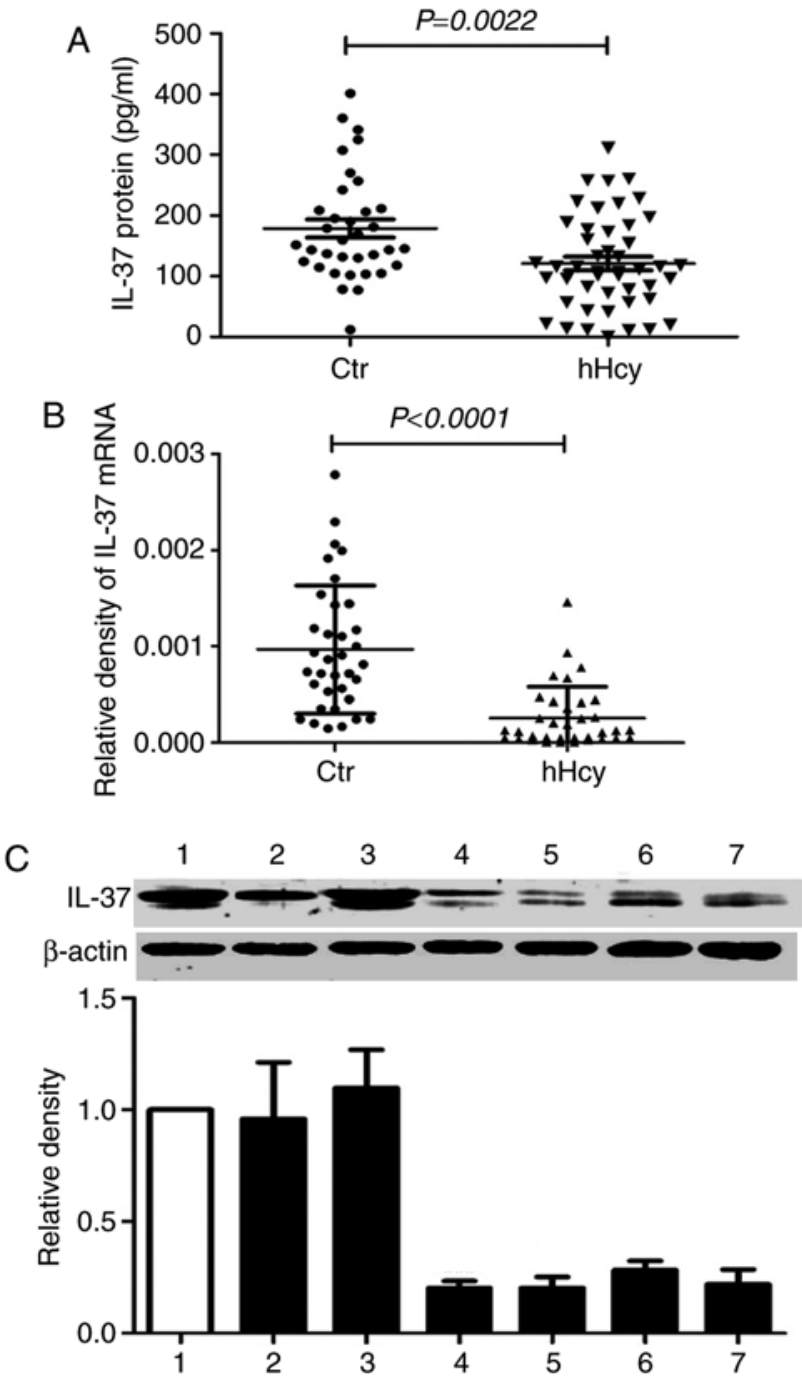

Figure 1. IL-37 expression in patients with hHcy. (A) IL-37 levels in serum was determined via ELISA. (B) mRNA expression levels of IL-37 in PBMCs as determined via reverse transcription-quantitative PCR analysis. (C) Protein expression levels of IL-37 in PBMCs as determined via western blotting. Lanes 1-3, normal controls; lanes 4-7, patients with hHcy. The error bars represented replicate measurements of the same samples in Fig. 1C. Ctr healthy individuals; hHcy, hyperhomocysteinemia; IL-37, interleukin 37; PBMCs, peripheral blood mononuclear cells.

effect on cell viability identified for $\leq 30 \mu \mathrm{mol} / 1$ Hcy compared with the control group $(\mathrm{P}>0.05)$. Cell viability was significantly inhibited by 23.3 and $47.8 \%$ when cells were treated with 60 and $120 \mu \mathrm{mol} / 1 \mathrm{Hcy}$, respectively ( $\mathrm{P}<0.01$; Fig. $3 \mathrm{~A})$.

$\mathrm{LDH}$ levels are an indicator of cell injury and were detected in the present study. Hcy led to the release of LDH, which is associated with cell damage. After $293 \mathrm{~T}$ cells were stimulated by Hcy $(30,60$ and $120 \mu \mathrm{mol} / \mathrm{l})$ for $24 \mathrm{~h}, \mathrm{LDH}$ levels in the supernatants were elevated. LDH levels were significantly increased by 1.8 times $(60 \mu \mathrm{mol} / 1 \mathrm{Hcy})$ and 3.0 times (120 $\mu \mathrm{mol} / 1$ Hcy), respectively ( $\mathrm{P}<0.01$; Fig. 3B).

Inflammation is another essential aspect of cell injury by Hcy $(1,2)$. In the present study, Hcy treatment promoted the secretion of the inflammatory cytokines, IL-1 $\beta$, IL-6 and tumor necrosis factor- $\alpha$ (TNF- $\alpha$ ). After 293T cells were stimulated with Hcy $(30,60$ and $120 \mu \mathrm{mol} / \mathrm{l})$ for $24 \mathrm{~h}$, the levels of IL-1 $\beta$ significantly increased by $2.1-, 3.5$ - and

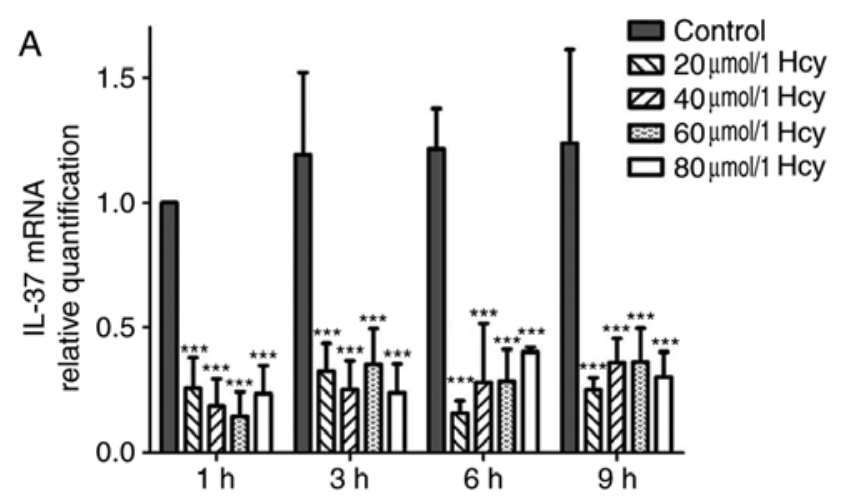

B Hcy $\begin{array}{llllllllll}0 & 10 & 20 & 30 & 40 & 50 & 60 & 70 & 80 & \mu \mathrm{mol} / 1\end{array}$
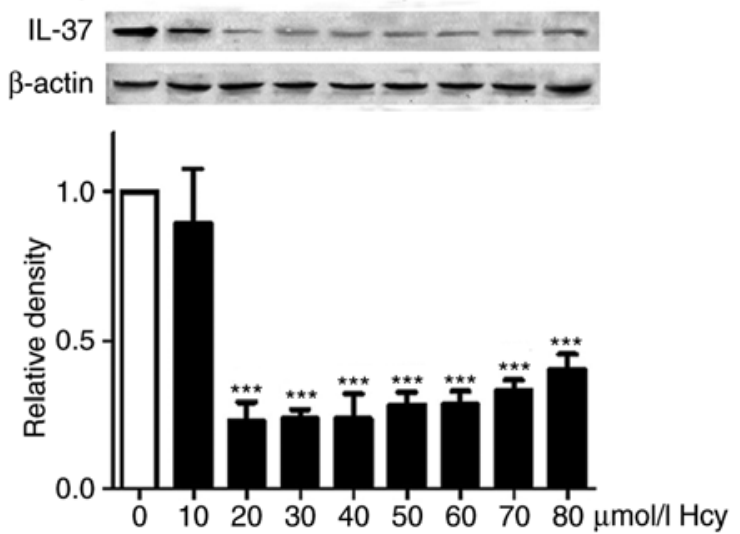

Figure 2. IL-37 expression is downregulated by Hcy in PBMCs. Exogenous Hcy was used to treat PBMCs prior to detection of the expression levels of IL-37. Cells in the control group were treated with PBS. (A) mRNA expression levels of IL-37. (B) Protein expression levels of IL-37. ${ }^{* * * *} \mathrm{P}<0.01$ vs. control (treated with PBS). Hcy, homocysteine; IL-37, interleukin 37; PBMCs, peripheral blood mononuclear cells.

4.4-fold, respectively, compared with those of the control group (Fig. 3C). The levels of IL-6 in the supernatants of cells stimulated by Hcy were increased 2.8-, 3.8- and 5.7-fold, respectively (Fig. 3D). The levels of TNF- $\alpha$ markedly increased by 1.9-, 3.2- and 4.3-fold, respectively, compared with those of the control group (Fig. 3E). Overall, the expression levels of IL-1 $\beta$, IL- 6 and TNF- $\alpha$ were increased with increasing Hcy stimulation.

IL-37 expression restores the proliferation ability of 2937 cells impaired by Hcy. First, the transfection efficiency of IL-37 plasmid was determined via RT-qPCR analysis. IL-37 mRNA was increased $>90,000$-fold, compared with that of $293 \mathrm{~T}$ cells transfected with pcDNA3.1 plasmid (data not shown). The results indicated that the gene transfection was successful and that the background expression of IL-37 was very low normally. In the following experiment, cells of the control group were treated with Hcy and were transfected with pcDNA3.1 plasmid. In the IL-37 group, cells were treated with Hcy and were transfected with pcDNA3.1/IL-37-eGFP plasmid. The cell viabilities of control cells were $95 \pm 3.2,75 \pm 5.1$ and $55 \pm 3.3 \%$, respectively, whereas the cell viabilities of the IL-37 group were $96 \pm 3.1,85 \pm 5.0$ and $78 \pm 3.2 \%$, respectively, following treatment with Hcy (30, 60 and $120 \mu \mathrm{mol} / \mathrm{l})$ for $24 \mathrm{~h}$ (Fig. 4A). The results revealed that IL-37 overexpression attenuated the inhibitory effects of Hcy on cell viability. 

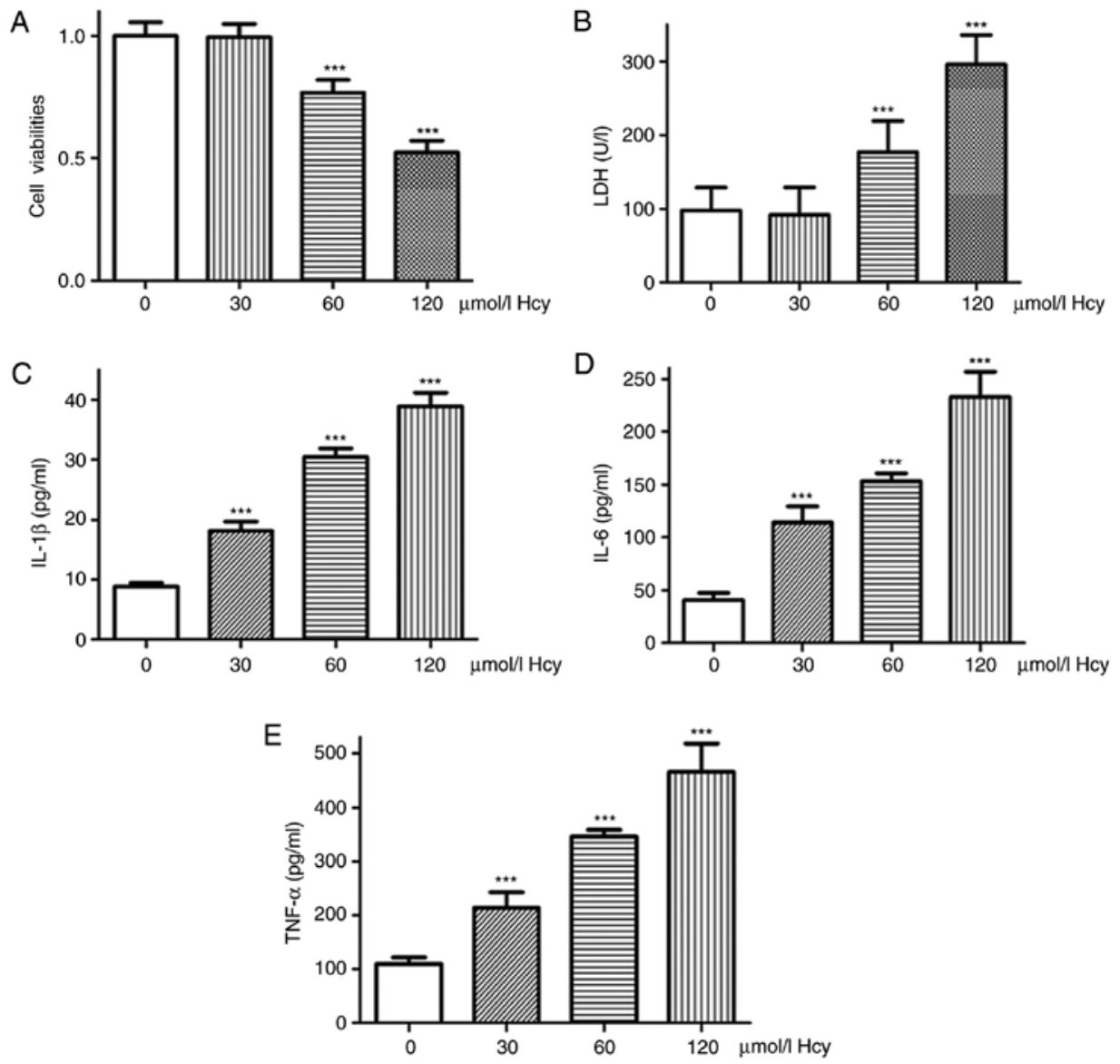

Figure 3. Effects of Hcy on cell viability, LDH release and expression of proinflammatory cytokines in 293T cells. (A) Effects of Hcy on cell viability. (B) Effects of Hcy on LDH release. (C) Effects of Hcy on the levels of the proinflammatory cytokine IL-1 $\beta$. (D) Effects of Hcy on the levels of IL-6. (E) Effects of Hcy on the levels of TNF- $\alpha$. Cells in the control group were treated with PBS. ${ }^{* * *} \mathrm{P}<0.01$ vs. control. Hcy, homocysteine; IL, interleukin; LDH, lactate dehydrogenase; TNF- $\alpha$, tumor necrosis factor- $\alpha$.

IL-37 expression inhibits Hcy-induced LDH release in 2937 cells. IL-37 functions in the protection of cell injury $(18,19)$. LDH release by the IL-37 group was decreased by 2.7 , 15.8 and $32.2 \%$, respectively, compared with the control group following treatment with Hcy $(30,60$ and $120 \mu \mathrm{mol} / \mathrm{l})$ for $24 \mathrm{~h}$ (Fig. 4B).

IL-37 decreases the levels of secreted inflammatory cytokines in the supernatant induced by Hcy in $293 \mathrm{~T}$ cells. Following treatment with Hcy $(30,60$ and $120 \mu \mathrm{mol} / \mathrm{l})$ for $24 \mathrm{~h}$, the expression levels of IL-1 $\beta$ in the supernatants of cells overexpressing IL-37 were downregulated markedly by $27.1,49.7$ and $60.4 \%$, respectively, compared with those of the $293 \mathrm{~T}$ cells in the control group (Fig. 4C).

Similarly, the expression levels of IL-6 in cell supernatants of the IL-37 group were markedly suppressed by $55.8,55.0$ and $69.4 \%$, respectively, compared with those of the control group (Fig. 4D). The expression levels of TNF- $\alpha$ in cell supernatants of the IL-37 group were suppressed markedly by 40.3, 45.7 and 54.4\%, respectively (Fig. 4E).

\section{Discussion}

IL-37 is a novel anti-inflammatory cytokine which is frequently abnormally expressed in a variety of inflammatory diseases (15-17). hHcy is an independent risk factor for a variety of inflammation-associated diseases, including atherosclerosis, coronary heart disease, chronic nephritis, stroke and neurodegeneration (1-10). However, the expression and the role of IL-37 in these hHcy-associated diseases remain unclear. Studies have demonstrated that IL-37 is highly expressed in patients with inflammatory bowel disease, rheumatoid arthritis, chronic hepatitis B, contact dermatitis, green barre syndrome and systemic lupus erythematosus (17-20). The present study focused mainly on IL-37 in mild hHcy as there were numerous abnormal factors in severe Hcy, so that the association between Hcy and IL-37 could not be clearly investigated. The levels of IL-37 in serum and the expression levels of IL-37 in PBMCs were markedly decreased in patients with mild hHcy. According to a previous study, IL-37 is highly expressed in atherosclerotic diseases, and IL-37 decreases rapidly following treatment with atorvastatin (22). Ji et al (23) reported that levels of plasma IL-37 were elevated in patients with acute coronary syndromes. In addition, animal studies have demonstrated that IL-37 can be used in the treatment of atherosclerosis $(22,23)$. High IL-37 is usually due to inflammation stimulation, but elevated IL-37 may exert a reverse effect and inhibit the inflammation. Thus, high IL-37 signals the body's resistance to disease and is helpful to treat disease. However, these studies have not examined the level of Hcy, and the association between Hcy and IL-37 $(22,23)$. In the inflammation pathway, Hcy promotes the secretion of inflammatory 

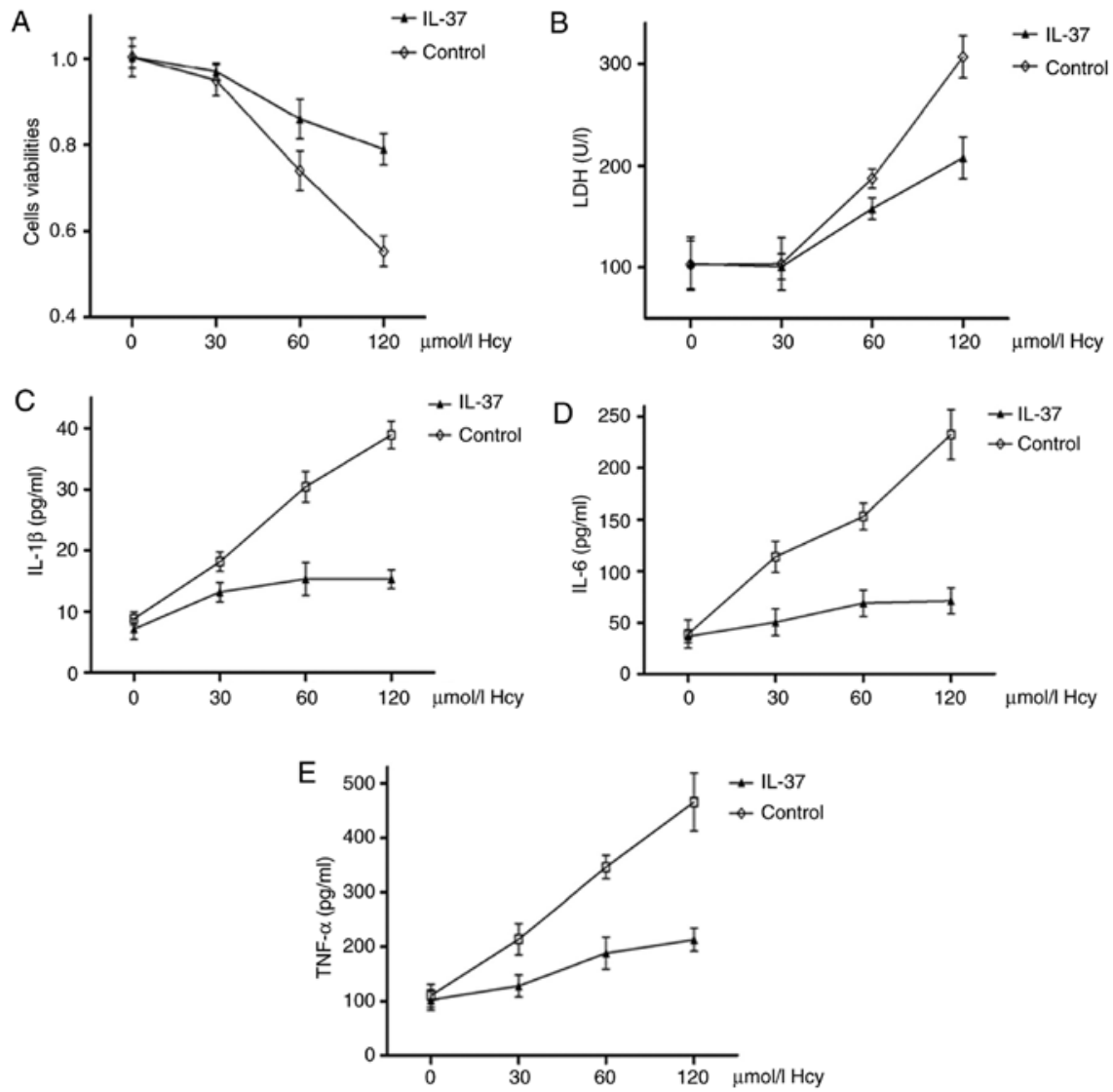

Figure 4. IL-37 expression protects cells from biological injury by Hcy. (A) Effects of IL-37 on the proliferation of 293T cells. (B) Effects of IL-37 on LDH release. (C) Effects of IL-37 on the levels of the proinflammatory cytokine IL-1 $\beta$. (D) Effects of IL-37 on the levels of IL-6. (E) Effects of IL-37 on the levels of TNF- $\alpha$. Control group cells were treated with Hcy and transfected with pcDNA3.1 plasmid. IL-37 group cells were treated with Hcy and transfected with pcDNA3.1/IL-37-eGFP plasmid. Hcy, homocysteine; IL, interleukin; LDH, lactate dehydrogenase; TNF- $\alpha$, tumor necrosis factor- $\alpha$.

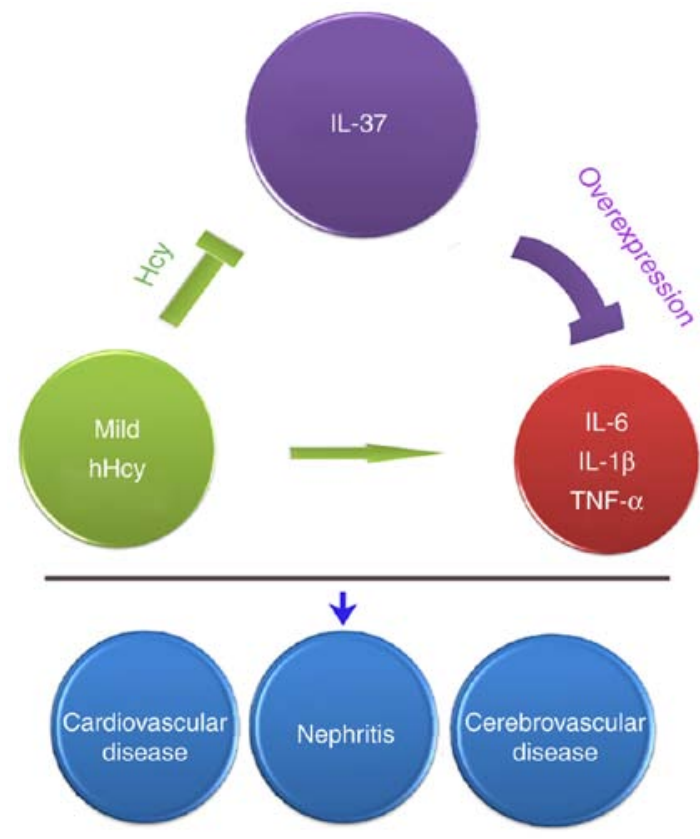

Figure 5. IL-37 is suppressed by Hcy and may protect cells from biological injury by hHcy. IL-37 is suppressed by Hcy and may protect cells from biological injury by hHcy. Mild Hcy could induce the release of inflammatory factors including IL-6, IL-1 $\beta$ and TNF- $\alpha$. Hcy also inhibit IL-37 expression to promote inflammation and causes kinds of diseases. Thus, overexpressing IL-37 can inhibit inflammation induced by Hcy and protect cells from Hcy-induced injury. hHcy, hyperhomocysteinemia; Hcy, homocysteine; IL, interleukin; TNF- $\alpha$, tumor necrosis factor- $\alpha$. cytokines, including macrophage inflammatory protein-1, monocyte chemoattractant protein-1, IL-1, IL-6, TNF- $\alpha$ and adhesion molecules, in smooth muscle cells, endothelial cells and mononuclear cells (26). However, as an important novel molecule involved in inflammatory responses, the expression and biological function of IL-37 in patients with hHcy remain unknown. It was speculated that, in the early stages of the diseases, the downregulation of IL-37 in patients with hHcy may lead to overexpression of inflammatory cytokines and cell injury, and thus induced the uncontrolled inflammation, leading to severe diseases. However, the mechanism underlying the downregulation of IL-37 in hHcy is unclear.

In order to further understand the mechanism of downregulation of IL-37 expression in hHcy, PBMCs and 293T cells were selected to perform Hcy stimulation experiments. hHcy can lead to atherosclerosis, coronary heart disease and other cardiovascular diseases which involve the injury of vascular endothelial cells. Renal diseases, such as nephrotic syndrome and chronic nephritis caused by Hcy are closely associated with renal epithelial cells (1-4). Therefore, 293T cells were used in the present study.

First, the downregulation of IL-37 was due to the slightly elevated Hcy which was associated with mild hHcy. Previous studies have mostly focused on the downstream anti-inflammatory effect of IL-37 $(15,16)$, and few studies have investigated the upstream regulation of IL-37. In a previous study (16), IL-37 has been reported to be upregulated in the 
majority of inflammatory diseases; however, in the present study, IL-37 was downregulated in mild Hcy. To the best of our knowledge, the effect of Hcy on IL-37 has not been studied yet. Mild but not severe hHcy resulted in decreased expression levels of IL-37 in PBMCs and 293T cells. HHcy is defined as serum Hcy levels $>15 \mu \mathrm{mol} / \mathrm{l}$; severe hHcy has been defined as Hcy $>100 \mu \mathrm{mol} / 1$.

In the present study, 'mild' hHcy means Hcy $<100 \mu \mathrm{mol} / 1$ in the cell culture supernatant and, considering that it is so different for the in vitro study compared with the in vivo study, an additional concentration 120 umol/1 was also used in the present study to help verify the effects of Hcy. It was hypothesized that, at the early stage of disease, IL-37 was downregulated. The downregulation of IL-37 may further promote uncontrolled inflammation.

Second, the effect of Hcy on cells was investigated. Hcy can promote the release of inflammatory factors in macrophages, monocytes, vascular endothelial cells and glial cells $(27,28)$. Zanin et al (28) used Hcy to treat rat macrophages, and revealed that the expression of IL-1 $\beta$ in cells and the supernatant was increased. Additionally, the NF- $\mathrm{BB}$ and ERK signaling pathways are activated by Hcy (28). This suggests that Hcy activates the inflammatory signaling pathways leading to the release of inflammatory mediators. Han et al (29) reported increased expression of the P65 protein, intercellular adhesion molecule 1 and IL-6 in endothelial cells stimulated with Hcy. In their study, Hcy also increased the levels of reactive oxygen species and activated the $\mathrm{NF}-\kappa \mathrm{B}$ pathway. In the present study, Hcy stimulation resulted in the inhibition of cell viability, and triggered the release of LDH and high expression of inflammatory cytokines, which was consistent with previous studies $(28,29)$.

Finally, the regulatory significance of IL-37 expression on cell injury and cellular inflammation induced by Hcy was explored. Overexpression of IL-37 could alleviate Hcy-induced cell viability inhibition and cell damage. Overexpression of IL-37 also significantly inhibited the expression of inflammatory cytokines such as IL-1, IL- 6 and TNF- $\alpha$. The association between IL-37, and inflammatory cytokines and cell injury has been studied in other diseases previously. Nold et al (15) reported that IL-37 decreases lipopolysaccharide-induced proinflammatory release. McNamee et al (30) demonstrated that increased IL-37 expression decreased IL-1 $\beta$ and TNF- $\alpha$ levels in the colon tissue of mice with colitis. The present study revealed that IL-37 reduced cell injury and inflammation induced by Hcy (Fig. 5). The findings suggested that treatment with IL-37 in the early stages of the disease may be able to block disease progression and mitigate disease damage.

In conclusion, IL-37 levels in the peripheral blood of patients with mild hHcy were noticeably lower. The expression levels of IL-37 were downregulated by Hcy stimulation, and IL-37 protected cells from inflammatory injury by Hcy treatment (Fig. 5). Therefore, IL-37 may have a protective role in mild hHcy, and be clinically meaningful for the prevention and treatment of Hcy-associated diseases.

\section{Acknowledgements}

Not applicable.

\section{Funding}

The present study was funded by the Science and Technology Plan Project of Guangdong Province (grant no. 2016A020215147), Natural Science Foundation of Guangdong Province, China (grant no. 2018A030307026), Medical Science and Technology Research Fund of Guangdong Province (grant no. A2017605), Scientific Research Foundation of Guangdong Medical University (grant no. Z2014002), Training Program of Innovation and Entrepreneurship for Undergraduates (grant nos. 201510571011 and 201510571056), Fund of the School of Laboratory Medicine of Guangdong Medical University, the Medical and Health Technology Plan Project of Special Fund for Economic and Technological Development of Longgang District, Shenzhen, PR. China (grant no. 20160606173542416) and National Natural Science Foundation of China (grant no. 81302244).

\section{Availability of data and materials}

The datasets used and/or analyzed during the current study are available from the corresponding author on reasonable request.

\section{Authors' contributions}

ZC conceived and supervised the study. ZC and SW designed the experiments. SW, ZH, HW, WL, SH, JZ, RL and ZL performed the experiments. SW, WL, ZL and ZC analyzed the data. SW and $\mathrm{ZC}$ wrote the manuscript and made manuscript revisions.

\section{Ethics approval and consent to participate}

The experiments were approved by the Science and Education Section of Eighth People's Hospital of Dongguan City. Written informed consent was obtained from all participants for the use of their samples in the present study.

\section{Patient consent for publication}

Not applicable.

\section{Competing interests}

The authors declare that they have no competing interests.

\section{References}

1. Zhao J, Chen H, Liu N, Chen J, Gu Y, Chen J and Yang K: Role of hyperhomocysteinemia and hyperuricemia in pathogenesis of atherosclerosis. J Stroke Cerebrovasc Dis 26: 2695-2699, 2017.

2. Jakubowski H: Homocysteine modification in protein structure/function and human disease. Physiol Rev 99: 555-604, 2019.

3. Rong D, Liu J, Jia X, Al-Nafisee D, Jia S, Sun G, Li Y, Lu W, $\mathrm{Li} \mathrm{H}$, Zhang $\mathrm{H}$, et al: Hyperhomocysteinaemia is an independent risk factor for peripheral arterial disease in a Chinese Han population. Atherosclerosis 263: 205-210, 2017.

4. Cao C, Hu J, Dong Y, Zhan R, Li P, Su H, Peng Q, Wu T, Lei L, Hang X, et al: Gender differences in the risk factors for endothelial dysfunction in Chinese hypertensive patients: Homocysteine is an independent risk factor in females. PLoS One 10: e0118686, 2015.

5. Xie D, Yuan Y, Guo J, Yang S, Xu X, Wang Q, Li Y, Qin X, Tang G, Huo Y, et al: Hyperhomocysteinemia predicts renal function decline: A prospective study in hypertensive adults. Sci Rep 5: 16268, 2015. 
6. Suszyńska-Zajczyk J, Utyro $\mathrm{O}$ and Jakubowski $\mathrm{H}$ Methionine-induced hyperhomocysteinemia and bleomycin hydrolase deficiency alter the expression of mouse kidney proteins involved in renal disease. Mol Genet Metab 112: 339-346, 2014.

7. Petras M,TatarkovaZ,Kovalska M,MokraD,DobrotaD,Lehotsky J and Drgova A: Hyperhomocysteinemia as a risk factor for the neuronal system disorders. J Physiol Pharmacol 65: 15-23, 2014.

8. Ashjazadeh N, Fathi M and Shariat A: Evaluation of homocysteine level as a risk factor among patients with ischemic stroke and its subtypes. Iran J Med Sci 38: 233-239, 2013.

9. Kamat PK, Vacek JC, Kalani A and Tyagi N: Homocysteine induced cerebrovascular dysfunction: A link to Alzheimer's disease etiology. Open Neurol J 9: 9-14, 2015.

10. Nazef K, Khelil M, Chelouti H, Kacimi G, Bendini M, Tazir M, Belarbi S, El Hadi Cherifi $M$ and Djerdjouri B. Hyperhomocysteinemia is a risk factor for Alzheimer's disease in an Algerian population. Arch Med Res 45: 247-250, 2014.

11. Han L, Wu Q, Wang C, Hao Y, Zhao J, Zhang L, Fan R, Liu Y, Li R, Chen Z, et al: Homocysteine, ischemic stroke and coronary heart disease in hypertensive patients. Stroke 46: 1777-1786, 2015.

12. Durgaprasad R, Akkulagari V, Kasala L, Velam V and Sarma P: GW27-e0552 Novel single nucleotide polymorphisms identified in Exon-10 of Human coagulation factor V gene in patients with pulmonary embolism. J Am Coll Cardiol 68: C48-C48, 2016

13. Mazza A, Cicero AF, Ramazzina E, Lenti S, Schiavon L, Casiglia E and Gussoni G: Nutraceutical approaches to homocysteine lowering in hypertensive subjects at low cardiovascular risk: A multicenter, randomized clinical trial. J Biol Regul Homeost Agents 30: 921-927, 2016.

14. Wang CY, Chen ZW, Zhang T, Liu J, Chen SH, Liu SY, Han LY, Hui ZH and Chen YM: Elevated plasma homocysteine level is associated with ischemic stroke in Chinese hypertensive patients. Eur J Intern Med 25: 538-544, 2014

15. Nold MF, Nold-Petry CA, Zepp JA, Palmer BE, Bufler P and Dinarello CA: IL-37 is a fundamental inhibitor of innate immunity. Nat Immunol 11: 1014-1022, 2010.

16. Boraschi D, Lucchesi D, Hainzl S, Leitner M, Maier E, Mangelberger D, Oostingh GJ, Pfaller T, Pixner C, Posselt G, et al: IL-37: A new anti-inflammatory cytokine of the IL-1 family. Eur Cytokine Netw 22: 127-147, 2011.

17. Luo Y, Cai X, Liu S, Wang S, Nold-Petry CA, Nold MF, Bufler P, Norris D, Dinarello CA and Fujita M: Suppression of antigen-specific adaptive immunity by IL-37 via induction of tolerogenic dendritic cells. Proc Natl Acad Sci USA 111: 15178-15183, 2014

18. Li Y, Wang Y, Liu Y, Wang Y, Zuo X, Li Y and Lu X: The possible role of the novel cytokines il-35 and il-37 in inflammatory bowel disease. Mediators Inflamm 2014: 136329, 2014
19. Ye L, Jiang B, Deng J, Du J, Xiong W, Guan Y, Wen Z, Huang K and Huang Z: IL-37 Alleviates rheumatoid arthritis by suppressing IL-17 and IL-17-triggering cytokine production and limiting Th17 cell proliferation. J Immunol 194: 5110-5119, 2015.

20. Zhao PW, Jiang WG, Wang L, Jiang ZY, Shan YX and Jiang YF: Plasma levels of IL-37 and correlation with TNF- $\alpha$, IL-17A and disease activity during DMARD treatment of rheumatoid arthritis. PLoS One 9: e95346, 2014.

21. Ye L, Ji L, Wen Z, Zhou Y, Hu D, Li Y, Yu T, Chen B, Zhang J, Ding L, et al: IL-37 inhibits the production of inflammatory cytokines in peripheral blood mononuclear cells of patients with systemic lupus erythematosus: Its correlation with disease activity. J Transl Med 12: 69, 2014.

22. Conti P, Lessiani G, Kritas SK, Ronconi G, Caraffa A and Theoharides TC: Mast cells emerge as mediators of atherosclerosis: Special emphasis on IL-37 inhibition. Tissue Cell 49: 393-400, 2017.

23. Ji Q, Zeng Q, Huang H, Shi Y, Lin Y, Lu Z, Meng K, Wu B, Yu K, Chai M, et al: Elevated plasma IL-37, IL-18, and IL-18BP concentrations in patients with acute coronary syndrome. Mediat Inflamm 2014: 165742, 2014.

24. Wu B, Meng K, Ji Q, Cheng M, Yu K, Zhao X, Tony H, Liu Y, Zhou Y, Chang C, et al: Interleukin-37 ameliorates myocardial ischaemia/reperfusion injury in mice. Clin Exp Immunol 176: 438-451, 2014

25. YeZ, Wang C, Tang J,Zhou Y, Bai L, Liu Y, Kijlstra A and Yang P: Decreased interleukin-37 expression in Vogt-Koyanagi-Harada disease and upregulation following immunosuppressive treatment. J Interferon Cytokine Res 35: 265-272, 2015.

26. Livak KJ and Schmittgen TD: Analysis of relative gene expression data using real-time quantitative PCR and the 2(-Delta Delta C(T)) method. Methods 25: 402-408, 2001.

27. Asanuma Y, Oeser A, Stanley E, Shintani A and Stein CM: The effect of homocysteine on the production of inflammatory cytokines. Clin Pharmacol Ther 75: P49, 2004.

28. Zanin RF, Bergamin LS, Morrone FB, Coutinho-Silva R, de Souza Wyse AT and Battastini AM: Pathological concentrations of homocysteine increases IL- $1 \beta$ production in macrophages in a P2X7, NF-kB, and erk-dependent manner. Purinergic Signal 11: 463-470, 2015.

29. Han S, Wu H, Li W and Gao P: Protective effects of genistein in homocysteine-induced endothelial cell inflammatory injury. Mol Cell Biochem 403: 43-49, 2015.

30. McNamee EN, Masterson JC, Jedlicka P, McManus M, Grenz A, Collins CB, Nold MF, Nold-Petry C, Bufler P, Dinarello CA and Rivera-Nieves J: Interleukin 37 expression protects mice from colitis. Proc Natl Acda Sci USA 108: 16711-16716, 2011. 